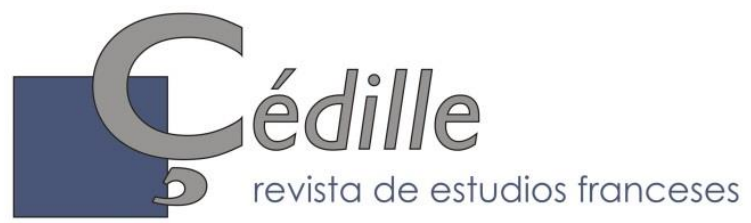

ISSN: 1699-4949

$n^{0} 18$ (otoño de 2020)

Monografías 11

Epistemocrítica: análisis literario y saber científico

Amelia Gamoneda Lanza \& Francisco González Fernández, editores científicos

\title{
La prosa científica de Pierre Teilhard de Chardin como texto sonoro: límites y cobordismos
}

\author{
Marta CuRESES \\ Universidad de Oviedo \\ cureses@uniovi.es \\ ORCID: 0000-0002-4184-4133
}

\begin{abstract}
Resumen
La creación artística en los años de vanguardia transgredió la delimitación tradicional entre formas y géneros. La teoría de cobordismos de René Thom, modelo de variedades que presentan el límite común de una misma variedad, sirve para explicar el proceso que eleva a rango literario un enunciado científico. En la frontera de poesía y ciencia, se analiza como ejemplo la obra Dilatación fonética del compositor Agustín González Acilu, concebida durante sus años de vida en París, sobre un texto en francés de Pierre Teilhard de Chardin.
\end{abstract}

Palabras clave: Poema en prosa, Texto científico, Composición, René Thom, Agustín González Acilu.

\section{Résumé}

La création artistique pendant les années d'avant-garde a transgressé la délimitation traditionnelle entre formes et genres. La théorie des cobordismes de René Thom, modèle de variétés qui présentent la limite commune d'une même variété, permet d'expliquer le processus qui élève au rang littéraire un énoncé scientifique. Aux confins de la poésie et de la science, il est question d'analyser l'exemple de l'œuvre Dilatation phonétique du compositeur Agustín González Acilu, conçue pendant les années qu'il avait vécues à Paris, sur un texte en français de Teilhard de Chardin.

Mots clé : Poème en prose, Texte scientifique, Composition, René Thom, Agustín González Acilu.

\begin{abstract}
Artistic creation during the avant-garde years transgressed the traditional delimitation between forms and genres. René Thom's cobordisms theory, a model of varieties sharing a common one, helps us in explaining the process for raising a scientific statement into a literary condition. At the border of poetry and sciencie, we consider here as an example González Acilu's composition Dilatación fonética, a work written during his years in Paris, on a French text by Pierre Teilhard de Chardin.
\end{abstract}

\footnotetext{
*Artículo recibido el 25/02/2020, aceptado el 25/10/2020.
} 
Keywords: Prose poem, Scientific text, Composition, René Thom, Agustín González Acilu.

\section{Introducción}

Georges Bataille concluye las últimas páginas de L'Érotisme manifestando que toda existencia, en lo concerniente a los hombres, está particularmente ligada al lenguaje, y el lenguaje nunca es independiente del juego entre lo prohibido y la transgresión. Podríamos haber comenzado con unas palabras de Jean-Jacques Ampère: «Les langues en général commencent par être une musique et finissent par être une algèbre», pues viene muy bien al planteamiento que aquí se expone. Precisamente esta es la cita elegida por Pierssens (1990: 55) ${ }^{1}$ para iniciar el cuarto capítulo de sus ensayos de epistemocrítica Savoirs à l'œuvre, dedicado a la lingüística de Mallarmé. El concepto de transgresión implica una norma, un rigor que de uno u otro modo se vulnera. La norma sugiere un código, y el código presupone un sistema de signos encaminado, en principio, al acto comunicativo.

Frente a las polémicas de los epistemólogos sobre la posible definición de lo que es y no es una ciencia, René Thom ${ }^{2}$ manifiesta que toda ciencia es, antes que nada, el estudio de una fenomenología, es decir, «los fenómenos que son objeto de una determinada disciplina científica aparecen como accidentes de formas definidas en un espacio dado, al que podemos llamar espacio substrato de la morfología que se estudia» (Thom, 1985: 9), y que en los ámbitos más generales, como la física o la biología, suele ser el espacio-tiempo. El matemático francés hace notar con cierto asombro que algunas disciplinas, sobre todo las ciencias del hombre, se preguntan cuáles son los hechos a estudiar antes de tener una descripción morfológica de su campo de estudio. Sus inquietudes en el ámbito de la topología algebraica, a la que se dedicó en los años cincuenta, le llevaron a interesarse por las llamadas variedades cobordantes, en el intento de averiguar cuándo dos variedades constituyen el límite común de una misma variedad, cuestión que, lejos de ser gratuita, reviste un tono filosófico:

Tenemos dos espacios, dos variedades diferentes, y se trata de algún modo de unirlas con una especie de deformación continua. El mejor procedimiento consiste en la construcción de un cobordismo entre las dos variedades. Con la ayuda de este tipo de ideas he desarrollado toda una técnica sobre las aplicaciones diferenciables, mediante la cual he podido

\footnotetext{
${ }^{1}$ La cita de Ampère procede de Histoire de la Littérature française au Moyen Âge comparée aux littératures étrangères (París, Just Tessier Libraire-Éditeur, 1841, p. 3).

Este artículo ha sido realizado en el marco de las actividades del Proyecto de I+D de Excelencia Inscripciones literarias de la ciencia: cognición, epistemología y epistemocrítica (ILICIA) del Ministerio de Economía y Competitividad (ref. FF12017-83932-P).

${ }^{2}$ René Thom (Montbéliard 1923 - Bures-sur-Yvette 2002).
} 
resolver, por lo menos técnicamente, si dos variedades son cobordantes, reduciéndolo a términos puramente algebraicos (Thom, 1985: 26).

En términos sencillos, un cobordismo (fig.1) para dos variedades $\mathrm{n}$-dimensionales $\mathrm{M}$ y $\mathrm{N}$ consiste en una variedad compacta de dimensión $(n+1)$ tal que los bordes de esa variedad consistan en la unión disjuntiva de las variedades dadas ${ }^{3}$. Es decir: cuando una variedad de dimensión (n) es la frontera de una variedad $(n+1)$. Heinz Hopf describía el cobordismo de Thom como una de esas construcciones aparentemente triviales de las que no se esperaban resultados significativos y que terminó siendo extremadamente fructífera. Los trabajos sobre el cobordismo hicieron merecedor a René Thom

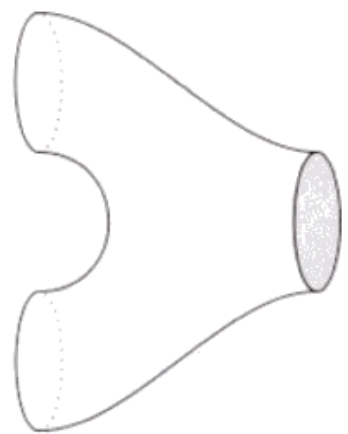

Fig. 1 de la medalla Fields, y su impulso de la rama de la matemática dedicada a la teoría de los sistemas dinámicos tuvo enseguida una prolongación filosófica que desembocó en el primer teorema de clasificación de las catástrofes elementales, proposición que unifica todos los hechos observables en la naturaleza «aussi bien du côté de la nature inorganique que dans la sphère biologique, ou même, atteignant la strate culturelle, à la base du langage humain» (Petitot, 1983: 4-64). Los estudios de René Thom en materia de semiolingüística estructural y cognitiva y su conexión con la teoría de las catástrofes fueron recibidos de manera desigual por los especialistas: su amigo Roman Jakobson fue siempre muy entusiasta, igual que los alemanes Hansjakob Seiler y Wolfganf Wildgen, el danés Per-Aager Brandt, Bernard Pottier en Francia o Umberto Eco en Italia, si bien la mayoría mostraron ciertas reservas. Encontramos ante este tema una situación similar a la que se produce frente a su teoría de la morfogénesis biológica. Para los especialistas en lingüística y semiótica Thom era una especie de meteorito del espacio cuyas teorías matemáticas no entendían (Petitot, 2015) y, por otro lado, los científicos que comprendían muy bien sus teorías matemáticas no tenían manera de entender las cuestiones semiolingüísticas que planteaba René Thom, cuyo máximo referente en la materia fue Lucien Tesnière ${ }^{4}$, a quien había conocido durante su etapa en Estrasburgo.

En La búsqueda de la lengua perfecta se pregunta Umberto Eco si no sería posible concebir una máquina capaz de generar todas las lenguas posibles a la manera de G. P. Harsdörffer y su propuesta en Mathematische und philosophische Erquickstunden (1651) donde plantea un juego de generación automática de casi

\footnotetext{
${ }^{3}$ La noción de cobordismo se basa en los presupuestos de la clasificación topológica planteada por Poincaré y Pontryagin. Véase una definición de este término, no reconocido en el Diccionario de la Lengua Española de la Real Academia, en https://www.universalis.fr/dictionnaire/cobordisme.

4 Tesnière (Mont-Saint-Aignan 1893 - Montpellier 1954) no llegó a ver publicada su obra Éléments de Syntaxe structurale (1959), que recoge su teoría sintáctica a partir de stemmas, representación gráfica precursora de la gramática generativa de Chomsky.
} 
cien mil palabras en alemán, incluidas aquellas que no existen, mediante combinatoria. Y refiriéndose a la postura de Valéry respecto al analysis situs o topología en su proyecto de topografía mental, muestra que la conexión entre cobordismo y topología no es privativa de René Thom. De hecho ha sido sugerida por diversos especialistas, entre ellos Judith Robinson (1962: 70):

La valeur de la topologie pour l'analyse de l'esprit réside dans le fait qu'elle permet d'étudier, en faisant abstraction de toute notion de quantité et de mesure, les rapports de contact et de continuité entre les points et les espaces qui les contiennent.

En el prólogo titulado «Épistémologie et Linguistique» que Thom escribe para el texto de Wildgen De la grammaire au discours. Une approche morphodinamyque, señala el particular estatuto que, entre todas las ciencias, tiene la lingüística pues, en efecto, entre todas «elle offre cette particularité d'être rigoureusement inutile» (Wildgen, 1999: 1). Aunque la explicación es compleja, fundamentalmente se refiere al hecho de que la transmisión de una lengua de padres a hijos se realiza por un proceso básicamente instintivo, a diferencia de lo que sucede en las «sciences dures». En este segundo tipo de ciencias la celebridad del hallazgo se asocia al nombre del descubridor -el teléfono de Bell, el radio de Curie, el átomo de Bohr o la vacuna de Pasteur -, mientras que en la lingüística hay dificultad para proceder de manera similar, pues los grandes lingüistas deben su celebridad, no a los hechos, sino «à des constructions philosophico-théoriques que la posterité, le plus souvent, a condensées en une brève formule. Il semble donc que ce soit au niveau d'une analyse abstraite de leur œuvre que sont jugés ces auteurs» (Wildgen, 1999: 1) ${ }^{5}$. En este cruce de caminos, el mestizaje epistémico y metodológico entre disciplinas ha dado lugar a debates que, con intención claramente proactiva, resuelven abandonar la conquista de territorio ajeno, un pacto de respeto mutuo, en favor de un trabajo en la frontera (Schwartz y Berti, 2018). Una frontera que no separa: plantea la posibilidad de intercambios que interesan a las partes.

Los límites de música y ciencia no existen como escisión entre campos de conocimiento independientes. El sentido de relación intrínseca entre sus respectivos ámbitos de acción puede auxiliarse, por ejemplo, de los escritos sobre la historia de la ciencia de William Whewell, donde se hace uso de la invención del término «colligation of facts» a fin de coordinar hechos e ideas en un contexto dialéctico apropiado, definiéndolo como una parte de la formación de nuestro conocimiento que podemos aplicar a todos los casos en los que, mediante nuestro intelecto, establecemos una conexión entre fenómenos que se presentan ante nuestros sentidos. El conocimiento de esas conexiones, acumulado y

5 Cita los casos de «l'innere Sprachform de W. von Humboldt, la ternarité de Peirce, le structuralisme jakobsonien, l'arbitraire du signe saussurien, le générativisme de Chomsky ». 
sistematizado, es Ciencia (Whewell, 1984: 206) ${ }^{6}$. Y aún añade otro término más preciso, «consilience of inductions», que emerge cuando una inducción obtenida de un género de hechos coincide con otra inducción obtenida de una clase distinta, algo así como una prueba de la veracidad teórica del supuesto. Una inducción no es la simple suma de hechos coligados: los hechos no se unen, se contemplan desde un nuevo punto de vista ${ }^{7}$.

Ahora bien, tal y como recoge René Thom en Esquisse d'une Sémiophysique. Physique aristotélicienne et Théorie des Catastrophes, «l'expérience première, en toute réception des phénomenes, est la discontinuité. Mais la discontinuité présupose le continu» (Thom, 1988: 17). Se trata, por tanto, de fenómenos que en matemática implican cambios bruscos o discontinuidad. Este es en esencia el pilar de la teoría de las catástrofes, que irrumpe en la escena científica y filosófica internacional en 1972 con la clamorosa aparición de su libro Stabilité structurelle et morphogénèse, suscitando un debate teórico que vino a ser el inicio de una ruptura epistemológica:

Un survol de cette singulière conjoncture montre qu'elle est l'effet de l'intrusion brutale de mathématiques fondamentales dans des régions réputées non formalisables et traditionnellement voueés à la langue naturelle. Comme si la séparation tranchée entre sciences quantitatives exactes et sciences descriptives «anexactes» se trouvait soudain remise en cause dans ses principes mêmes ${ }^{8}$.

Filosofía y epistemología de la matemática encuentran así un representante idóneo en la figura de René Thom: «La philosophie, ou l'épistémologie de la mathématique au sens large a vu l'émergence d'une figure singulière, qui mérite une place à part dans ce panorama» (Salanskis, 2019). Cuando Claude LéviStrauss se detiene en «Les mathématiques de l'homme» para describir los valores tópicos de la lengua, tanto para las ciencias sociales como humanas, enfatiza en la composición de ésta a base de elementos discontinuos, los fonemas, añadiendo que, tras las aportaciones de Trubetzkoy, Jakobson, Benveniste, Sapir, Bloomfield, Hjemslev o Sommerfelt al estructuralismo de Saussure, esta disciplina asentada en el carácter discontinuo de sus componentes microscópicos se rige por leyes que ofrecen un grado de rigor enteramente comparable a las leyes de correlación que observamos en las ciencias exactas y naturales (Lévi-Strauss, 1970). La construcción de aparatos que, como el voder ${ }^{9}$, fueron concebidos para poder realizar la síntesis de la palabra y condujeron a la formalización teórica de

\footnotetext{
${ }^{6}$ «We may apply this term to every case in which, by an act of the intellect, we establish a precise connection among the phenomena which are presented to our senses. The knowledge of such connections, accumulated and systematized, is Science».

${ }^{7}$ « Is not the mere sum of facts which are colligated. The Facts are not only brought together but seen in a new point of view » (Whewell, $1984: 257$ ).

${ }^{8}$ Una explicación de este debate puede leerse en el artículo de Jean Petitot (2019).

${ }^{9} \mathrm{El}$ voder fue presentado en 1949 por el matemático e ingeniero Claude Shannon.
} 
sus métodos, refuerza la argumentación e interés posterior de esos procesos de análisis.

La síntesis concatenativa de sonidos vocales y su aplicación mediante técnicas como la que emplea el vocoder (voice-coder, o codificador de voz) no llegan a su aplicación en el arte sonoro hasta los años setenta, de manera que el empleo de aparatos como el antiguo espectrógrafo resultaban sumamente modernos en la década de los sesenta, sobre todo en España, donde, ciertamente, nadie lo empleaba como instrumento auxiliar de la escritura musical. Un compositor navarro, Agustín González Acilu (Alsasua 1929) será pionero en plantear un trabajo en la frontera de la música y la fonética a través de la prosa científica de Teilhard de Chardin, tratada como texto literario, y la sonoridad de la lengua francesa en la que está escrito el texto original, analizado espectrográficamente en el laboratorio del Consejo Superior de Investigaciones Científicas (CSIC). La prosa de Teilhard de Chardin, integrada en el planteamiento inédito de Acilu al emplearla como base sonora de la partitura de su obra Dilatación fonética (1966), y el procedimiento compositivo propio del análisis fonético-fonológico del texto, resultan bastante más trascendentes de lo que en su momento se entendió, que no fue gran cosa. Mediante el análisis de la musicalidad contenida en la tonología hablada, no cantada, de la lengua francesa, quedaba de manifiesto la posibilidad de generar el rigor que respalda el carácter científico de los presupuestos constructivos de la obra. Aún faltaban años para que los nombres más sobresalientes del panorama internacional, como Pierre Boulez, Luigi Nono, Karlheinz Stockhausen, pero sobre todo Luciano Berio -discípulo de Giorgio Ghedini, igual que Acilu en su etapa romana- se aproximasen a un planteamiento similar.

En cuanto al origen matemático de la composición musical, el número siempre ha estado presente, desde Pitágoras hasta hoy: series de Fibonacci, estructuras fractales, física teórica y teoría de cuerdas, principios de combinatoria y de estadística no agotan las fuentes de construcción sonora a lo largo de la historia.

La conocida sentencia de Teilhard de Chardin - «Lo infinitamente grande, lo infinitamente pequeño, lo infinitamente complejo»- presidía la página web de una conocida revista de ciencia, tecnología y sociedad que el 31 de octubre de 2002 abría con el titular «La catástrofe de la desaparición de René Thom», fallecido cinco días antes. El compositor Pascal Dusapin se inspiró en sus teorías para alguna de sus obras, como Cycle des sept formes y Loop. Salvador Dalí concibió su Tratado de escritura catastrofeiforme después de haber conocido a Thom en 1978. Algunas de sus obras de los años ochenta están ideadas a partir de la teoría de catástrofes: La cola de Milano, que en su título alude a la tercera de las siete catástrofes elementales (fig. 2$)^{10}$. También El rapto topológico de Europa

\footnotetext{
${ }^{10}$ Las siete catástrofes elementales son: el pliegue, la cúspide, la cola de milano, la mariposa y las tres umbílicas (elíptica, hiperbólica y parabólica). Los nombres de las cuatro primeras provienen
} 
(Homenaje a René Thom), que muestra una vista aérea de un mapa de Europa fracturado por una grieta en una superficie alabeada sobre la que ha escrito una ecuación de Thom a modo de leyenda explicativa (fig.3).

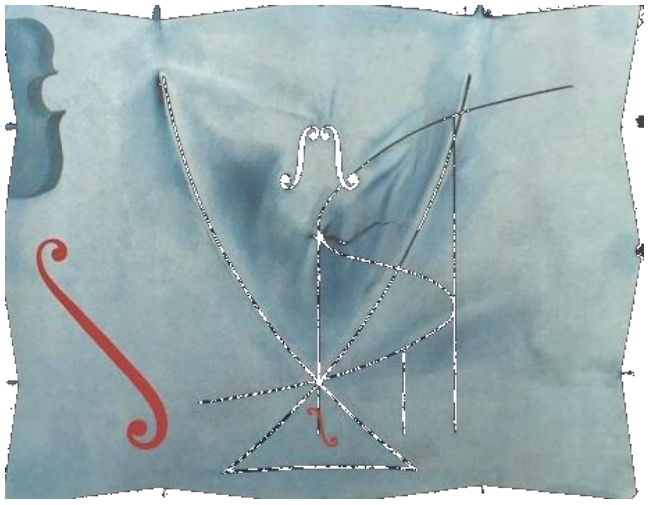

Fig. 2

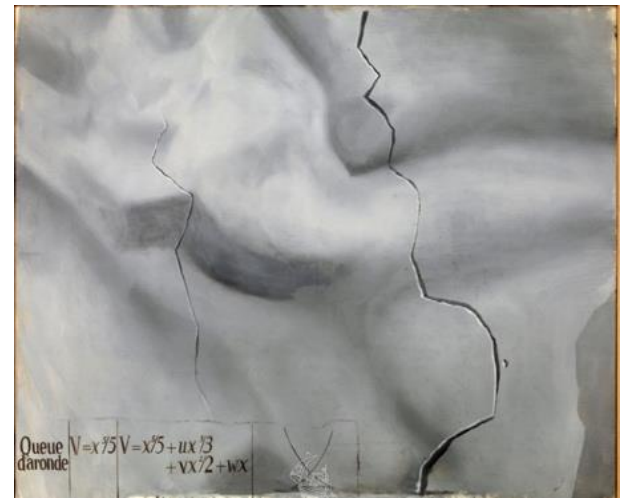

Fig. 3

\section{Desactivación de perspectivas formales binarias}

Es posible que en la morfogénesis del poema en prosa se encuentre una de las causas de la disolución de fronteras, no solamente entre poesía y prosa, sino entre géneros cuyos contenidos responden a categorías distintas. Las características enunciadas por Suzanne Bernard (1959) -brevedad, unidad orgánica, gratuidad- han quedado en una teoría cuyos hilvanes históricos, siendo de puntada firme, no son el único patrón posible. Ciertamente se parte de los fundamentos más conocidos sobre el tema, como la reivindicación de Baudelaire en la dedicatoria «À Arsène Houssaye» $(1920)^{11}$ de ambicionar, siquiera en sueños, el milagro de una prosa poética, musical, «sin ritmo ni rima», con la ductilidad que permita al texto oscilar de lo flexible a la rigidez más contundente para así «pour s'adapter aux mouvements lyriques de l'âme, aux ondulations de la rêverie, aux soubresauts de la conscience» (Baudelaire, 1869: 1). Aloysius Bertrand queda designado allí como iniciador del género, como referencia en este intento literario de Baudelaire. En este estudio, tomado como imprescindible en muchos otros posteriores, sería conveniente añadir a la relación musical de los simbolistas con Wagner su intenso intercambio con Claude Debussy. Citemos solamente algunas obras del compositor francés, como Cinq poèmes de Baudelaire sobre textos de Les Fleurs du mal, Claire de lune, Fantoches, Fêtes galantes, Mandoline, Trois mélodies, En sourdine, Pantomime y Ariettes oubliées, todas estas últimas sobre textos de Paul Verlaine, y muy especialmente Prélude à l'aprés-midi d'un faune, poema sinfónico inspirado por el poema homónimo de Stéphane Mallarmé, a quien está dedicada la partitura considerada por muchos

de los rasgos visuales que presentan los gráficos que las describen; las tres restantes, más difíciles de visualizar, llevan nombres matemáticos.

11 Texto publicado en La Presse el 26 de agosto de 1862 e incluido en Baudelaire (1920): Petits poëmes en prose ou Le Spleen de Paris (1920 [1862]). 
especialistas génesis de la música moderna. Además de otras páginas célebres, de su trabajo conjunto surgió la partitura de Anna la bonne, canción hablada («chanson parlée») inspirada en un poema de Poe traducido al francés por Mallarmé.

Deben añadirse, finalmente, algunas otras referencias que apuntalan la génesis de una nueva visión, como las que aporta Michel Sandras en el «avantpropos» de Lire le poème en prose: «Un grand amateur de poésie, Henri Deluy, affirme que le poème en prose lui procure des plaisirs inquiétants» (apud Deluy 1989: 7), ejemplificando acto seguido la línea casi invisible que separa «poème en prose» $\mathrm{y}$ «prose poétique» con un fragmento de «Revêrie au Lido» en las Mémoires d'outre-tombe de Chateaubriand. La introducción de Henri de SaintDenis al Préface de Cromwell de Victor Hugo (1971: 14) establece con claridad el valor formal del célebre texto:

Tal vez lo que más nos interesa hoy del prólogo de Cromwell es la visión que el autor nos da de un espectáculo total, según él lo concibe, y aquí hemos de limitar esta expresión al aspecto puramente literario. Espectáculo total en tanto que aboga por la destrucción de los géneros establecidos.

Poniendo como ejemplos al boticario de Romeo y Julieta, las brujas de Macbeth, los sepultureros de Hamlet o al Rey Lear, Maurice Souriau (1897: 231) subraya las palabras de Victor Hugo con las que en el Préface se cuestiona la distinción de géneros: «Shakespeare, ce dieu du théâtre, en qui semblent réunis, comme dans une trinité, les trois grands génies caractéristiques de notre scène: Corneille, Molière, Beaumarchais. On voit combien l'arbitraire distinction des genres croule vite devant la raison et le goût».

Otras reflexiones sobre el poema en prosa se remontan a la oratoria sublime de Bossuet, o a François de Malherbe, autor de Larmes de Saint Pierre, para quien no existían diferencias entre prosa y poesía, excepto el metro, o a varios prosistas franceses del siglo XVII, como se menciona en el estudio de María Victoria Utrera (1999: 29) dedicado a la teoría del poema en prosa:

Ya desde Francisco de Sales puede apreciarse en Francia la tendencia a un prosa poética más musical, más lírica y emotiva que elocuente, tendencia que se concreta en la literatura francesa en la obra de Fénelon, Télémaque (1699), donde es fácil percibir un deseo de libertad rítmica, de suave sonoridad que se corresponde con el propósito de su autor de una eficaz renovación de la prosa que iba a tener consecuencias importantes en la literatura francesa y en la génesis de una nueva prosa poética de más alto vuelo ${ }^{12}$.

Parece así probada la imposibilidad de establecer límites a la hora de considerar si un texto presenta o no los rasgos pertinentes (y excluyentes de

${ }^{12}$ Utrera cita como referencia el estudio de Albert Cherel La prose poétique française (París, L'artisan du Livre, 1940). 
cualquier otra posibilidad) que definen su condición poética, científica o prosaica. Contraviniendo la práctica secular que tantos debates ha generado desde, al menos, el Renacimiento, todos esos textos están acreditados para integrarse en una obra musical: la distinción formal entre géneros, abolida desde la anulación del binomio forma y esencia, suspende la necesidad de asociación con estructuras previamente establecidas. En el estudio de las formas el concepto de estabilidad es fundamental, hasta el punto que la estabilidad estructural es condición de existencia y conocimiento. En el ámbito de la matemática el proceso se desarrolla de forma análoga: la estabilidad estructural es un concepto clave en la interpretación de toda disciplina, excepto en la mecánica cuántica, como se explica en los modelos de la morfogénesis: un proceso $[\mathrm{P}]$ es estructuralmente estable si una leve variación de las condiciones iniciales conduce a un proceso $\left[\mathrm{P}^{\prime}\right]$ isomorfo a $[\mathrm{P}]$ en el sentido de que una pequeña perturbación sobre el espaciotiempo -un $\varepsilon$-homeomorfismo en geometría- transforma de nuevo el proceso $\left[\mathrm{P}^{\prime}\right]$ en $\left[\mathrm{P}^{\prime \prime}\right]$ (Thom 1971). La interrupción de un proceso por catástrofe no es, por tanto, sino discontinuidad en su desarrollo. René Thom llevó su teoría de las catástrofes más allá del ámbito matemático, al dominio de la filosofía, de la lingüística y de la poesía.

Las reflexiones de Poe (1846: 163-167) sobre filosofía de la composición aclaran algunos extremos: por ejemplo, que lo que solemos considerar un poema extenso no es más que una sucesión de poemas cortos, o lo que es lo mismo, una sucesión de efectos poéticos breves. O que, de todas las consideraciones a tener en cuenta a la hora de componer un texto (un relato, un poema), la más importante es el efecto que se pretende causar. Estas y otras teorías suscitaron una incredulidad sincrónica a su recepción ${ }^{13}$. Estudios coetáneos como los de Kahn (1925) y Bremond (1926), ofrecen visiones distintas de un mismo panorama. Como ha precisado Robert Guiette (1964: 845), Kahn diferencia dos tipos de poema en prosa: «L'un plastique, sobre, stricte, apportée par Bertrand ; l'autre chantante et musicale, innovée par Baudelaire ${ }^{14}$. Por su parte, el historiador y jesuita de la escuela moderna de filosofía Henri Bremond, profesor en Moulins, Saint-Étienne y Villefranche-sur-Saône, donde tuvo como alumno a Teilhard de Chardin, y autor de textos contemplados con autoridad en su momento -Les deux musiques de la prose (1924), La poésie pure (1926), además de su magna obra Histoire littéraire du sentiment religieux en France (1916-1936) $)^{15}$ - cree que los «modernes théoriciens» de la poesía pura -Poe, Baudelaire, Mallarmé y Valéry-

\footnotetext{
${ }^{13}$ Un desarrollo preciso del tema puede leerse en Esperando a Gödel. Literatura y matemáticas de Francisco González Fernández (2012: 109-118).

${ }^{14}$ Incide en este aspecto en su «Préface sur le vers libre», donde, a propósito de la armonía y ritmo propio de la prosa, señala como fundamental «de permettre à tout poète de concevoir en lui son vers ou plutôt sa strophe originale, et d'écrire son rythme propre et individuel» (Kahn, 1897: 28).

${ }^{15} \mathrm{Su}$ autoridad viene respaldada sobre todo por su calidad de miembro de la Académie Française desde 1923. Su Histoire littéraire, en once volúmenes, fue publicada asimismo por la Académie Française
} 
no son los peligrosos innovadores que a veces se cree: «Nous pouvons, certes, les soupçonner d'hérésie sur quelques points de détail, et je ne m'en prive pas; mais pour le fond de la doctrine, ils continuent une tradition assez vénérable» (Brémond 1926: 15). Respecto a la musicalidad del poema, queda convertida en finalidad puesto que la poesía consiste en llegar a captar «pour les orchestrer délicieusement, les ressources musicales du langage». Su definición del poeta, que «n'est qu'un musicien entre les autres», le lleva a identificar poesía y música «mais, la musique pure ne paraissant pas moins mystérieuse que la poésie, je me demande si ce n'est pas là definir l'inconnu par l'inconnu» (Brémond, 1926: 23). $\mathrm{Su}$ postura limita la definición y está orientada a un sentido casi místico como finalidad que se aparta del pensamiento de todos los autores que ha citado antes:

S'il en faut croire Walter Pater, tous les arts aspiraient à rejoindre la musique. Non, ils aspirent tous, mais chacun par les magiques intermédiaires qui lui sont propres, - les mots ; les notes; les couleurs; les lignes - ils aspirent tous à rejoindre la prière (Brémond, 1926: 27).

En realidad, lo que Walter Pater ha dicho es que todo arte aspira a la condición de la música ${ }^{16}$, mientras que Bremond relega esta cualidad, la musicalidad de la palabra, por resultarle obvia, como señala Guillermo Carnero (2010: 59) apuntando a un sentido fundamentalmente sonoro: «Al fin y al cabo, el último estadio de la pureza [poética] puede consistir en elaborar un instrumento de comunicación exclusivamente fonosemántico, como última alternativa al silencio del que hablaba Bremond $\gg{ }^{17}$. Distinto es lo que sostiene Steiner en la introducción a Lecturas, obsesiones y otros ensayos: cualquier pensamiento sobre las formas del significado tiene que vérselas con la música. En su dualidad, como ciencia y como arte, algo se resiste al análisis cuando se trata de la segunda acepción:

Aunque los avances en neurofisiología de la visión, en la comprensión de los códigos simbólicos, en la sociología de los géneros estéticos, han aumentado y refinado grandemente nuestra respuesta a las artes plásticas, es justo decir que nuestra percepción de la naturaleza de la música, nuestros análisis de las formas en que la música llega a «poseer» y afectar nuestra psique, no han progresado decisivamente desde Platón (Steiner, 1990: 25).

Jacques Paquin (2008) señala en su reflexión sobre la inclusión de textos científicos en un poema una primera cuestión previa: «Que devient un texte scientifique (voire un microtexte) quand il est importé dans et par le discours poétique ?». Entre los ejemplos que ofrece para dilucidar si se trata de un cambio de estatus o pura retórica cita el poema «Fonction de Mandelbrot» de Renaud

\footnotetext{
16 «All art constantly aspires towards the condition of Music », una máxima procedente de su ensayo «The School of Giorgione » (The Fortnightly Review, 1877), posteriormente incluido en Pater (1988: 86).

${ }^{17}$ Un desarrollo del planteamiento se encuentra en Carnero (1989).
} 
Longchamps, preguntándose si podría considerarse un objeto fractal: tras largas disquisiciones, la respuesta es múltiple y abierta. En el ámbito de la composición musical, la teoría de fractales no solamente serviría para ponerle música a los principios de Benoît Mandelbrot, sino que hace ya muchos años que es un sistema de construcción sonora. En el contexto francés, desde sus orígenes la música ha combinado las tendencias más sublimes del melodismo con un espíritu definido por el sentido racionalista y científico de la Escuela de París y del autor del Ars Nova, el teórico, poeta y compositor parisino Philippe de Vitry; también por Guillaume de Machaut, creador de magníficos motetes isorrítmicos.

De las muchas teorías formuladas sobre la música como lenguaje interesan ahora aquellas que sitúan el código sonoro en paralelo con el lingüístico desde el punto de vista evolutivo, con un discurso cambiante, vivo en definitiva. Y, primordialmente, aquellas que han tratado de establecer semejanzas entre los cambios más profundos que esos códigos han experimentado en manos de los principales movimientos de vanguardia, tanto literaria como musical, y los derivados de ellas bajo el signo de estéticas diversas a lo largo del pasado siglo $\mathrm{XX}$, entre ellos los que ofrece la matemática. Las propuestas de los matemáticos de nuestro tiempo, grandes arquitecturas de forma y significado, han superado la necesidad de un discurso narrativo privativo desarrollando lenguajes propios tan articulados y elaborados como los del discurso verbal.

Los experimentos realizados con sonorizaciones no convencionales de textos, es decir textos no musicalizados sino tratados fonéticamente, nos remiten al trabajo pionero de Pierre Henry (1927 - 2017) a partir de su integración en el Groupe de Recherche Musicale (GRM) de la RTF creado en París por el científico, compositor y filósofo Pierre Schæffer en 1948. De su extensa obra pueden citarse aquí Fragments pour Artaud, encargo de Alain Trutat para la radio $^{18}$, Christal/Mémoire, Hörspiel d'après Marcel Proust ${ }^{19}$, y especialmente Maldoror/Feuilleton. Feuilleton radiophonique d'après Les Chants de Maldoror de Lautréamont en 50 episodes, sobre una adaptación de Pierre Henry de los Chants de Maldoror ${ }^{20}$. Podrían sumarse varias obras de Henri Pousseur (19292009) como Flexions hermétiques pour Baudelaire y L'École d'Orphée. Leçons d'Enfer sobre textos de Rimbaud, entre otras, y que, con excepción de Phonèmes pour Cathy dedicada a Cathy Berberian y compuesta sobre textos de Paul Claudel, no encajan en el epígrafe «creación sonora experimental», teniendo en cuenta que las técnicas del sprechgesang y el sprechstimme connaturales a la Escuela de Viena se normalizaron en su empleo desde mediados del siglo pasado.

\footnotetext{
18 Estrenado en Royan, el 21 de marzo de1970, con la voz de François Dufrêne y locutores anónimos.

${ }^{19}$ Obra radiofónica de tres horas de duración, ofrecida en la WDR de Colonia con las voces de Hanna Schygulla y Heinz Bennent.

${ }^{20} \mathrm{Su}$ emisión comenzó el 14 de febrero de 1993 en France Musique, todas las tardes desde esa fecha hasta completar cincuenta episodios.
} 
El procedimiento inverso, es decir, musicalizar un texto literario se encuentra en la morfogénesis de géneros como la chanson francesa de Janequin, la chanson borgoñona de Binchois y Dufay o el libro de madrigales de Philippe Verdelot y los madrigales del Renacimiento y primer Barroco que desembocaron en la ópera. De manera que, aunque la práctica consuetudinaria de varios siglos de historia -especialmente en el gran género de la música vocal, pero también en el canto de los padres de la iglesia- nos lleva por el camino que señala la estructura textual como pauta de organización sonora, existen muchos estudios dedicados a localizar y documentar el trasvase de estructuras musicales que han constituido la génesis de obras literarias. Son innumerables, de manera que sirva de ejemplo solamente un caso: Ulises de James Joyce, por haber sido de alguna manera una inspiración en la técnica que incide en el estudio tonológico de una lengua como única música, procedimiento que luego analizaremos, y porque fue objeto de numerosas reflexiones a partir del Omaggio a Joyce citado por Umberto Eco al inicio de Obra abierta:

Entre 1958 y 1959, yo trabajaba en la RAI de Milán. Dos pisos más arriba de mi despacho estaba el estudio de fonología musical, dirigido entonces por Luciano Berio. Pasaban por él Maderna, Boulez, Pousseur, Stockhausen; era todo un silbar de frecuencias, un ruido hecho de ondas cuadradas y sonidos blancos. En aquellos tiempos yo estaba trabajando en Joyce y pasábamos las veladas en casa de Berio, comíamos la comida armenia de Cathy Berberian y leíamos a Joyce. De allí nació un experimento sonoro cuyo título original fue Homenaje a Joyce, una especie de transmisión radiofónica de cuarenta minutos que se iniciaba con la lectura del capítulo II del Ulises (el llamado «de las Sirenas», orgía de onomatopeyas y aliteraciones) en tres idiomas: en inglés, en la versión francesa y en la italiana. Sin embargo, después, dado que el propio Joyce había dicho que la estructura del capítulo era de fuga per canonem, Berio comenzaba a superponer los textos a manera de fuga, primero inglés sobre inglés, luego inglés sobre francés y así sucesivamente, en una especie de polilingüe y rabelaisiano fra Martino Campanaro (Eco, 1979: 1).

Joyce incrementa la musicalidad del undécimo capítulo en varias direcciones; la musicalización de su escritura se extiende hacia las aliteraciones, las onomatopeyas, pero además organiza la entrada y salida de los personajes a la manera de una fuga per canonem, tal como menciona Eco. La fuga contenida en la escena del bar Ormond asigna el «sujeto» al barman, la «respuesta» a Bloom, a Boylan el «contrasujeto»y destina otros elementos de la fuga al resto de personajes. Así lo señala el crítico y traductor Stuart Gilbert, autor de James Joyce's «Ulysses»: A Study (1930), que tuvo un papel importante en la corrección de la traducción de Ulises al francés realizada por Auguste Morel, y es citado como autoridad en la mayoría de estudios dedicados a la música contenida en esta 
obra. Entre ellos el de Zack Bowen, Musical Allusions in the Works of James Joyce: Early Poetry through Ulysses (1974), donde ya se señala que casi no existen referencias musicales en la obra de Joyce antes de Ulises y que la mayor parte de estudiosos del autor irlandés se han dedicado a las alusiones musicales en ella, muchas veces en forma de menciones esporádicas de líneas concretas de su prosa, con la indicación de la música -normalmente el texto que acompaña a una melodía- a la que pertenecen. Otros autores realizan una valoración más global de la estructura textual: Martin Ross ve todo el texto de Ulises como una composición musical, más que una obra literaria, Father Robert Boyle escribe «Ulysses as a Frustrated Sonata Form», Frederick Sternfeld remite a «Poetry and Music. Joyce's Ulysses», Vernon Hall indica dos referencias muy determinadas en «Joyce's use of Da Ponte and Mozart's Don Giovanni» y James Penny Smith a «Musical Allusions in James Joyce's Ulysses». Estas referencias están recogidas y estudiadas por el propio Bowen en «The Bronzegold Siresong: A Musical Analysis of the Sirens Episode in Joyce's Ulysses» (Bowen, 1995).

El texto seleccionado para el Omaggio a Joyce organizado en la sede del Laboratorio de Fonología Musical de Milán, el capítulo de las sirenas, es el que ha sido objeto de un mayor número de estudios, precisamente por la musicalidad intrínseca de su composición: «The Sirens Episode as Music: Joyce's Experiment in Prose Polyphony» (Lawrence Levin), «The Sirens at the Ormod Bar: Ulysses» (Stanley Sultan) o «The Search for The Song the Sirens Sang: Two Notes on the Sirens of Joyce's Ulysses» (Marian H. Kaplun) son algunas de las reflexiones más interesantes, que no agotan el repertorio sobre un tema del que Bowen es quizá el mayor experto. Su conocimiento e interés por la estructura musical presente en los textos de Joyce conduce a una revisión de estudios tan curiosos y meticulosos como los llevados a cabo por Mathew Hodgart y Mabel Worthigton, Song in the Works of James Joyce o Weldon Thornton, Allusions in "Ulysses", 21 .

La estética de la disonancia en Thema. Omaggio a Joyce (1959), de Luciano Berio, abre la puerta a las sonoridades fruto de la superposición de la lectura de un mismo texto en varias lenguas, se recrea en la imposibilidad semántica y traduce en sonidos electroacústicos una interpretación vocal pregrabada. La autoría del planteamiento, como construcción de un discurso no musical, se debe a Umberto Eco, a cuyas clases magistrales asiste González Acilu como alumno durante su estancia en Roma, una etapa que ya hemos abordado en un estudio anterior (Cureses, 2001: 49), donde citamos sus palabras:

La modernidad estaba en la calle, se respiraba en el ambiente, algo pasaba cuando cruzabas la frontera y descubrías la libertad, y empezabas a creer en ti mismo. Esto sólo lo puede entender la

${ }^{21}$ Los autores del primero de ellos realizan un listado de más de mil referencias a otras tantas melodías citando textualmente las frases, su número de página y obra, si bien no explican la función o significado de las mismas en el marco de los pasajes en que Joyce las ha ubicado; en el segundo, el completo listado confeccionado por Thornton incluye no solo la línea y número de página en el texto de Ulises, sino muchas citas de letras de canciones donde pueden localizarse la música. 
gente de mi generación. Umberto Eco abría horizontes mucho más interesantes que Karajan, que seguía en La Fenice dirigiendo sinfonías de Beethoven.

\section{La hipótesis en el pensamiento de Teilhard de Chardin y González Acilu}

Nuestro conocimiento empírico del mundo que nos rodea, sea en el ámbito científico o en el cotidiano, se fundamenta básicamente en conjeturas e hipótesis con distinto grado de verosimilitud. Emplear la hipótesis como herramienta de trabajo tiene cierta dosis de atracción intuitiva, ya que se ajusta bien al concepto común de la naturaleza científica (Barker, 1957). Compuesta en 1966, la partitura que lleva por título Dilatación fonética tiene su origen unos años antes, durante la estancia de su autor en París, cuando el interés por las distintas lenguas, las distintas variantes tonales, la musicalidad que, en definitiva, todas ellas poseen, animan a González Acilu en el propósito de componer una obra para voz y grupo instrumental cuyo motor será el propio texto, del que emanará el discurso musical. Premio Príncipe de Viana de Cultura, Doctor Honoris Causa por la Universidad de Navarra y dos veces Premio Nacional de Música, entre otras distinciones, los inicios de Acilu fueron difíciles. En 1962 obtiene por concurso-oposición la beca de la Diputación Foral de Navarra para ampliar estudios en París. Esta beca le permitirá residir en el Colegio de España de la capital francesa, una experiencia decisiva tratándose de su primera estancia de larga duración en el extranjero. Inaugurado en 1935 y situado en el Boulevard Jourdan de la Cité Internationale Universitaire, el Colegio de España era en los años sesenta un crisol de intercambio cultural orientado al estímulo de las relaciones entre instituciones y centros de investigación de diverso signo. A su frente estaba Joaquín Pérez Villanueva, su director entre 1957 y 1968. Esta primera estancia -pues luego vendrían otras en Roma, Venecia o Darmstadt- como becario de la Institución Príncipe de Viana, es la más significativa en su etapa de perfeccionamiento. En París tiene oportunidad de asistir a los conciertos del Domaine Musical organizados por Pierre Boulez, así como a numerosas actividades a las que acude con sus grandes amigos pintores, Antonio Zarco y Manuel Alcorlo. La biblioteca del Colegio de España le da acceso a lecturas que determinarán la orientación de sus intereses en dos direcciones: la lingüística y la ciencia.

Pierre Teilhard de Chardin es el autor del texto elegido como base para elaborar la partitura de Dilatación fonética, texto que llega a sus manos a través de la lectura de un ejemplar de la revista Scientia. Las características de su pensamiento encajan en el entorno «lenguaje sonoro y ciencia» que se propone abordar Acilu. Una obra cuya sonoridad emanará de la propia tonología de la lengua francesa y que le permitirá convertir un texto científico en material artístico. En el estudio de Barthélemy-Madaule -cuya tesis doctoral consagró a las relaciones entre Bergson y Teilhard de Chardin- dedicado a «Les structures teilhardiennes de l'évolution», se plantean las fronteras que separan y al tiempo aproximan tres disciplinas fundamentales. Ciencia, religión y filosofía anticipan la 
disolución de fronteras, enuncian los cobordismos que definen sus respectivos contenidos:

On sait quelles confusions entre philosophie, science et religion illustrent les débats sur l'évolution depuis Lamarck et Darwin jusqu'à nos jours (inclus). Les exclure et les dépasser, ce n'est pas abolir de légitimes rapports; l'isolement des disciplines qui se sont indûment mêlées n'est pas une erreur moins grave que la pression abusive de l'une sur l'autre. D'une part, on ne confond qu'en dénaturant: la religion devient préjugé étouffant, la science prétentieuse, la philosophie religion déguisée. D'autre part, on n'isole qu'en sclérosant (Barthélemy-Madaule, 1963: 41).

Nacido el 1 de mayo de 1881 en Orcines, Château de Sarcenat, a siete kilómetros de Clermont-Ferrand, los estudios secundarios de Teilhard de Chardin transcurren en el colegio de padres jesuitas próximo a Villefranche-surBeaujolais. A los dieciocho años ingresa en la Compañía de Jesús y en 1906 se traslada a El Cairo, donde se inician sus investigaciones en paleontología. Después de la Segunda Guerra Mundial es nombrado director de investigación del Centre National de la Recherche Scientifique y se le ofrece un sillón en el Collège de France. Su pertenencia como Miembro de honor de The New York Academy of Sciences, de la Geological Society of America y del Institut de France da cuenta de la trascendencia de su trayectoria profesional. Los tres niveles de pensamiento fundamentales que pueden establecerse en su obra, la obra del especialista en paleontología, la obra fenomenológica y la obra mística o espiritual, están atravesados por el sentido científico que preside todas ellas, pese al cuestionamiento reiterado de algunos de sus planteamientos:

Un certain nombre de savants, de biologistes notamment reprochent à Teilhard de s'aventurer en tant que scientifique. Nous ne pouvons entrer dans ce débat épistémologique complexe, mais pour nous d'intérêt assez scolaire, de savoir si l'élucidation d'un sens de l'évolution biologique est ou non une démarche proprement scientifique (Escalere, 1967: 196).

La mayoría de sus estudiosos le muestran, no obstante, siempre al corriente de movimientos como la física teórica, la ciencia prerrelativista y precuántica, saberes que forman parte de su visión del mundo, de la weltanschauung teilhardiana. Su caso constituye «el primer logro francés de un uomo universale, el primer weltgeist auténticamente nacido de la tierra de Francia»(Cuénot, 1967: 545). Es bien conocido su perfil más lírico, el que se refleja en sus poemas-oraciones, donde con frecuencia sus textos remiten al macrocosmos (universo) y microcosmos (hombre) que hablan del origen pitagórico de estos pensamientos; un arco de alianza entre lo profano y lo sagrado presidido por el sentido musical que también impregna buena parte de su prosa, 
como reproduce Remo Vescia, presidente honorífico del Centre Européen Teilhard de Chardin, de una carta manuscrita del jesuita a una de sus primas:

Chaque œuvre poétique veut avoir son rythme propre, un rythme de tout l'ensemble, et un rythme des parties, et un rythme des paragraphes et des phrases... un rythme littéraire, vraiment senti et exprimé devrait ressembler à un morceau de musique, avec des nuances, des silences, des thèmes, une harmonie ensemble, une typographie spéciale, sa musicalité propre (Vescia, 2010).

$\mathrm{Su}$ obra como hombre de ciencia adquiere muchas veces el tono de prosa poética que le confiere seguramente su carácter visionario, en muchos sentidos, en una época en la que existen resistencias notables a sus teorías. Cuando Madeleine Barthélemy-Madaule redacta su tesis doctoral en 1963, el nombre de Teilhard de Chardin ya se ha incorporado a la historia de la filosofía en textos como los de Roger Garaudy (1959), pero también ha experimentado el descrédito, que se prolonga durante los años sesenta del siglo pasado a través de autores como Paul Ricœur, que se jacta de que Teilhard no le interesa nada, por un conjunto de complejas razones: reticencia ante lo que denomina esnobismo teilhardiano, su idealismo universitario, la antipatía de las nuevas izquierdas francesas hacia su persona, pero, sobre todo, por la gran pereza que le produce la necesidad de volver a pensar toda la filosofía por su causa (Cuénot, 1967). Quienes tienen contacto directo con su persona y su obra se sitúan más bien en otra línea: «Teilhard n'est pas seulement un savant, c'est un poète qui a la capacité rare de vision éclairante et d'expression incantatoire» (Vescia, 2010).

La hipótesis como herramienta de trabajo esencialmente dinámica es también la base sobre la que opera Teilhard de Chardin aprovechando sus rasgos de carga, que define, desde la primera página de Comment je crois (1969), como «axes de progression»o «lignes de pénétration». La pulcritud en el proceso de investigación favorece el desarrollo de la hipótesis, que aparece como una construcción médiatrice entre el acto de inspiración y la realización. De este planteamiento surge la práctica habitual en la obra completa de Acilu: encadenar hipótesis, a manera de eslabones de una cadena que representa el todo orgánico de su producción, pues cada nueva página parte de una hipótesis anterior que, de alguna manera, debe ser superada; una proposición tomada directamente de este pensador que revolucionó el ámbito religioso proponiendo la investigación científica como forma de adoración:

La fecondité d'une hypothèse, son pouvoir de «se développer indéfiniment», non seulement «sans contradiction interne», mais selon l'architecture la plus solide et la plus élégante, cette fécondité est fonction de ces axes, à la conjonction desquelles jaillira la plus haute hypothèse, celle qui soutiendra la vision toute entière. Ainsi, d'une part, chaque hypothèse est au centre d'un jaillissement éclairant et dispersif; mais, d'autre part, chaque hypothèse 
centrant autour d'elle la diversité des faits, elle-même centrée sur une hypothèse plus ample, peut être comprise, et l'ensemble avec elle, dans un puisant système convergent (Barthélemy-Madaule, 1967: 572).

De las tres posibles clases de hipótesis que Poincaré ofrece en La Science et l'Hypothèse -las verificables mediante la experiencia, las que sin inducirnos a error ayudan a fijar nuestro pensamiento, y las simples definiciones $\mathrm{o}$ convenciones que se presentan como mera apariencia- nos encontramos aquí con el segundo caso, siendo así que la hipótesis no solamente es necesaria «mais que le plus souvent il est légitime» (Poincaré, 1968: 24). Y si hacemos caso a $L a$ explicación científica de Richard Braithwaite (1965: 31), «no hay tragedia mayor que el asesinato de una hermosa hipótesis científica por un ejemplo discordante». Como proposiciones empíricas, no confinadas a campos limitados del espacio y del tiempo, su aplicación en la matemática nos permite una pluralidad de métodos para disponerlas en forma de sistema.

\section{Dilatación fonética: plan de trabajo y análisis}

Las condiciones de trabajo en Madrid tras la estancia en París se presentan difíciles para González Acilu. Ha perdido el contacto con la realidad de la situación que se vive en el contexto artístico español. Pese a ello, ya se ha trazado un plan que conduce sus pasos hacia la sede del Consejo Superior de Investigaciones Científicas. El material sonoro de su nuevo proyecto será el texto de Pierre Teilhard de Chardin: «Reflexions sur la valeur et l'avenir de la systématique». Conviene mencionar que no será esta la única ocasión en la que Acilu trabaje en el CSIC. Su Oratorio panlingüístico, obra por la que le fue concedido el Premio Nacional de Música en 1971, es otro de sus proyectos más ambiciosos en el contexto del análisis fonético de textos-base, en este caso un texto en euskera y castellano cuyo análisis espectrográfico fue supervisado por Luis Michelena y Ambrosio Zatarain, de la Real Academia Vasca de la Lengua. Por otra parte, sus obras posteriores, entre ellas Aschermittwoch (1968) -sobre un texto en alemán de Hans Magnus Enzensberger- y en la obra basada en el poema homónimo de Gerhard Rhüm, Hymnne an Lesbierinnen (1972) el tratamiento de los textos literarios experimenta el mismo proceso de transformación en material de investigación y se beneficia de los resultados alcanzados con su trabajo en lengua francesa.

Su trabajo sobre textos se ha desarrollado siempre en la frontera de la música y la fonética, con una percepción espacial muy próxima a la visión dinámica de Bergson, «le plus musicien des philosophes» ${ }^{22}$. Una noción incipiente del límite se encuentra en páginas tempranas, como Sucesiones superpuestas (1962), resultado de la confrontación tonalismo / atonalismo, técnica / estética, concepto / medio, materia / forma; en los confines de estas dicotomías se van

22 Madeleine Barthélemy-Madaule (1967: 17), recoge en su estudio las palabras de Bergson sobre su ascendente musical : «Mon père fut un compositeur et pianiste des plus distingués ». 
gestando los principios de una ontología sonora que dará lugar a Partita óntica (1987) o Variaciones ónticas $(1981)^{23}$. Entre estos binomios se encuentra asimismo la distinción entre Aesthetik (estética) y Kunstwissenschaft (ciencia del arte) que corresponde principalmente al debate alemán de principios del siglo pasado, pero de hecho «la estética siempre ha superpuesto y confundido ambos territorios» (Calabrese, 1985: 76), difuminando una vez más los contornos interdisciplinares.

Consecuencia de Dilatación fonética es la contraposición de la naturaleza física e histórica de la música, un enfrentamiento entre ambas naturalezas, sus límites y la potencial capacidad de transgresión de estos. Su dialéctica del límite va encaminada hacia un nuevo concepto que esboza la disolución de contornos. Se inicia así un proceso homólogo al desarrollo de la ontología del límite que Eugenio Trías expone en Los límites del mundo, donde este concepto experimenta una suerte de crisis por crecimiento, tal como se explica en las páginas dedicadas al debate sobre La filosofía del límite ${ }^{24}$ y coincidente con el sentido de dilatación que propone la obra de Acilu, esto es, una ampliación o crecimiento y no tanto anulación del mismo como formula Ortega en el ámbito de su Ensayo de la limitación, cuyos antecedentes explica Julián Marías en su edición de Meditaciones del Quijote ${ }^{25}$. A este planteamiento responde la trilogía del límite concebida por Acilu -Límites I, Límites II, Límites III (2009)- que pone de relieve la capacidad de indagación respecto a los límites de las fuerzas centrípetas y centrífugas que afianzan sus presupuestos estéticos.

Un periodo largo de consultas con diversos especialistas, en primer lugar en Pamplona y San Sebastián por la proximidad geográfica a sus orígenes, facilitan las primeras nociones generales de fonética y fonología: José María Satrústegui (1930-2003), antropólogo y etnógrafo navarro, miembro de número de la Real Academia de la Lengua Vasca y secretario de la misma en la época en la que Acilu trabajaba en Dilatación fonética; Luis Michelena (1915-1987), lingüista destacado entre las máximas autoridades sobre la lengua vasca y cuyo proyecto más ambicioso se materializó en el trabajo colectivo Diccionario de la lengua vasca; Fernando González Ollé, miembro de la Academia Navarra de la Lengua; Alfonso Irigoyen (1929-1996), lingüista, escritor, versolari y académico; José Antonio Arana Martija (1931-2011), académico, musicólogo y lingüista; Ambrosio Zatarain (1912-1999), ingeniero industrial y licenciado en Ciencias por la Universidad de Burdeos, exiliado, vivió en Pau, donde se formó en lingüística; Manuel Lecuona (1894-1987), lingüista, escritor, director de la Real Academia de la Lengua Vasca entre 1967 y 1970. Las referencias a la correspondencia y entrevistas mantenidas con ellos durante el proceso de creación de su obra se conservan en el archivo personal de Acilu.

${ }^{23}$ Una explicación más detallada se encuentra en Cureses (2011).

${ }^{24}$ Sus tres estudios, Lógica del límite, La edad del espíritu y La razón fronteriza constituyen la conocida trilogía del límite. Una visión global del tema se ofrece en Los límites del mundo (2000).

${ }^{25}$ Véase José Ortega y Gasset, Meditaciones del Quijote (1990). 
Finalmente, sería Antonio Quilis (1930-2003) quien proporcionase el soporte necesario a los entonces escasos conocimientos de Acilu para llevar a término su proyecto, comenzando por explicarle pormenorizadamente «El método espectrográfico. Notas de fonética experimental» ${ }^{26}$ (Navarro Tomás, 1960). La fonética y la dialectología fueron dos de las principales dedicaciones de Quilis en el Centro de Estudios Históricos de Madrid (CEH), cuya sección de Filología había encomendado tiempo atrás a Menéndez Pidal la misión de visitar en Francia, Suiza y Alemania centros universitarios pioneros en estudios de fonética. Aquellos estudios se prolongaron a lo largo de dos años, y en Montpellier tuvo ocasión de trabajar junto a Grammont y Millardet. Como resultado de sus logros, a inicios del siglo XX se instaló en el CEH un primer laboratorio de fonética «con un simple quimógrafo adquirido en París por don Pedro Blanco, miembro del Museo Pedagógico de Madrid, interesado en la nueva fonética experimental bajo los auspicios del laboratorio de Rousselot en el Collège de France» ${ }^{27}$, un espacio a todas luces escaso, hasta su traslado al Palacio del Hielo, en la calle Duque de Medinaceli, lugar designado como sede del Centro de Humanidades del Consejo Superior de Investigaciones Científicas. Sus aportaciones enlazan con otro de los nombres de referencia para Acilu, Tomás Navarro Tomás, introductor de la perspectiva acústica en los estudios de fonética española ${ }^{28}$, señalando la necesidad de tener presentes tanto los rasgos articulatorios como los acústicos, un planteamiento que, a su vez, Quilis conecta con las investigaciones desarrolladas por Bertil Malmberg en «Le problème du classement des sons du langage» $\mathrm{y}$ «Questions de méthode en phonétique syncronique» (Malmberg, 1952 y 1956).

En su texto dedicado al procedimiento de análisis fonético, entonces tan novedoso, señala Antonio Quilis como uno de los avances más importantes del método espectrográfico para la fonética el papel de las transiciones de los formantes en la percepción de las consonantes, tal y como había sugerido Pierre Delattre en «Les indices acoustiques de la parole» (1958), donde sintetizaba las principales investigaciones llevadas a cabo hasta ese momento sobre los rasgos acústicos de los sonidos. Toma asimismo como referencia el trabajo de Delattre «Un triangle acoustique des voyelles orales du français» (1948), pues en este último texto, a partir de los resultados acústicos obtenidos mediante el espectrógrafo, traza la figura pentagonal en la que sitúa las once vocales orales francesas con vistas al estudio de las relaciones acústicas y fisiológicas en las mismas. No obstante, lo decisivo para el tratamiento musical del texto de

\footnotetext{
${ }^{26}$ Un breve estudio publicado años atrás en la Revista de Filología Española que, no obstante, fue una iniciación fundamental para el compositor (Navarro Tomás, 1960).

27 Así lo define Tomás Navarro Tomás en su trabajo dedicado a la presencia de D. Ramón Menéndez Pidal en el CEH (Navarro Tomás, 1968-1969: 9).

28 En relación al trabajo desarrollado por Quilis (1930-2003) en este ámbito, M. J. Albalá, miembro del CSIC, ofrece una completa visión de su labor en la necrológica publicada en los Anales del Instituto de Estudios Madrileños XLIV, 2004,949-957.
} 
Dilatación fonética es lo que Quilis (1960: 427) aporta a propósito de la descomposición de los sonidos en armónicos:

Como ya sabemos, en virtud del teorema de Fourier, que cada uno de estos armónicos es un múltiplo del fundamental, el poder hallar el tono de este no es muy complicado. Esta es una de las principales ventajas del método espectrográfico. El armónico correspondiente a la línea melódica del fundamental aparece a simple vista; no hay más que aplicar la escala para averiguar su frecuencia. Para mayor exactitud en la medida, se pueden hacer distintas calas en los armónicos recordando siempre su propiedad de múltiplos de fundamental.

Desde el inicio del proceso de análisis llevado a cabo en las instalaciones del CSIC en Madrid la ayuda de Antonio Quilis fue determinante, no solo en una primera parte formativa y orientadora, sino en la propia realización del análisis espectrográfico que sirvió de partitura base para el desarrollo posterior del material analizado quimográficamente. Previamente a la realización del análisis, Quilis había recibido un complejo cuestionario elaborado por Acilu: una serie de preguntas encaminadas a trazar un puente entre aspectos estructurales de la composición y la perspectiva científica con la que se pretendía abordar el estudio fonético-fonológico del material textual de base. Siguiendo la descripción de Delattre de la lengua francesa -antériorité, mode croissant, forte tension articulatoire, égalité rythmique- Acilu trabaja el texto de Teilhard de Chardin exactamente igual que haría si se tratase de un texto de ámbito literario. Proporciona así un contexto artístico-musical al texto científico, dotándolo de una vida literaria, poética, en su declamación, teniendo en cuenta que esa declamación, incidiendo en la musicalidad intrínseca de la lengua en que está escrito, cambia el registro de su percepción de inmediato. Su planteamiento quiere distanciarse expresamente de la práctica habitual, buscando un nuevo enfoque a las relaciones música-texto que, por otra parte, presenta un carácter de continuidad en su trayectoria investigadora, casi puramente especulativa, puesto que poco antes ha trabajado ya en el ámbito instrumental con la microinterválica que desembocará en este análisis espectrográfico de textos. Este distanciamiento nos resulta familiar al leer las reflexiones de Thom:

Una gran parte de mis descubrimientos proceden de la pura especulación y se podrían catalogar como ensoñ mías. Acepto tal calificación, porque el acto de soñar ¿no es acaso una catástrofe virtual donde se inicia el conocimiento? En una época en que muchos científicos se dedican a calcular ¿no es deseable que algunos de ellos pudieran soñar? (apud Hayek, 2006).

En la obra que seguidamente analizaremos, Dilatación fonética, toda una síntesis de posibilidades fonéticas nos distancian de la semanticidad del texto, cubriendo una amplia gama de matices interpretativos, en ocasiones casi imperceptibles, pues el tratamiento que Acilu confiere al material-base de su obra (la 
prosa científica de Teilhard de Chardin), subraya los rasgos fonéticos ciñéndose al análisis riguroso de su entonación hablada. Estos aspectos estructurales están concebidos como parte de un sistema cohesionado mediante un procedimiento de extracción de potencialidades sonoras que auxilian al procedimiento constructivo, un sistema de trabajo que no procede del entorno compositivo musical. En su introducción a la Sistematización cognoscitiva, Rescher realiza una incursión en el campo de la metasistemática del conocimiento haciendo uso de una cita de Kant en Crítica de la razón pura, donde califica el arte de construir sistemas como «arquitectónico» (Rescher, 1981: 13), una tesis que pertenece al enfoque epistemológico caracterizado como coherentismo ${ }^{29}$. En este sentido conviene recordar que Acilu trabaja aquí, además de con presupuestos fonéticos y fonológicos, con principios de una rama de la física como es la acústica.

Dilatación fonética se presenta como una hipótesis de trabajo formulada por el compositor, un procedimiento que se convertirá en habitual en creaciones posteriores -la hipótesis como herramienta de trabajo- y de una hipótesis trata también el texto de Pierre Teilhard de Chardin, centrado en su importancia en relación al desarrollo de la ciencia, una serie de reflexiones sobre el valor y el futuro de la sistemática. El texto extraído, como antes se ha indicado, del artículo «Reflexions sur la valeur et l'avenir de la systématique» (Teilhard de Chardin, 1925: 15), dice así:

Loin d'être un accessoire pour la science, l'hypothèse est le but, l'âme et la vraie consistance des constructions scientifiques changeantes, fragiles, mais progressives comme la vie. Les bonnes hypothèses se modifient continuellement, mais dans un sens précis, suivant lequel elles se perfectionnent. Et au terme de cette évolution, elles passent au rang d'éléments définitifs destinés à figurer ultérieurement dans tout édifice représentatif du monde. Pierre Teilhard de Chardin ${ }^{30}$.

La primera cuestión que surge en el posicionamiento de Acilu frente al acto compositivo es la falta de congruencia en la relación entre la acentuación rítmica del texto y la interválica derivada del material sonoro a emplear, cuando este material procede del atonalismo: si el lenguaje posee polaridades derivadas de su entonación como principal característica, por qué forzar esa entonación a un mundo ajeno, ¿sería posible concebir elementos compositivos que se distanciasen tanto de la polaridad tonal como atonal? Este es el planteamiento que fundamenta el tratamiento del texto, presentado con carácter científico, bordeando lo literario en busca de una iconicidad que logrará mediante la sonoridad del propio texto. La selección de efectivos instrumentales que se unen a la voz solista obedece a un

${ }^{29}$ Escuela neohegeliana de Oxford.

${ }^{30}$ La firma del autor, Pierre Teilhard de Chardin, forma también parte del texto en la obra. En los ejemplos gráficos posteriores se observan varias erratas en el texto transcrito manualmente a la partitura. 
criterio de sonoridad monotímbrica, funcionalidad dirigida a centrar la atención del oyente en la microinterválica. La plantilla es la siguiente: viola de afinación normal, viola de afinación alterada $1 / 4$ de tono ascendente, violoncello de afinación normal y violoncello de afinación alterada $1 / 4$ de tono ascendente; la tesitura de la voz será de barítono. La colocación del grupo (fig.4) es fundamental para lograr los propósitos del autor: el objeto de los cuartos de tono obedece a la pretensión de disturbar en la medida de lo posible el principio físico-armónico, por ello los instrumentos deberán situarse intercalados entre sí según su distinta afinación. La materia sonora está producida por oposición en cuanto a su afinación, proporcionando un color adecuado a la estructura íntegra.

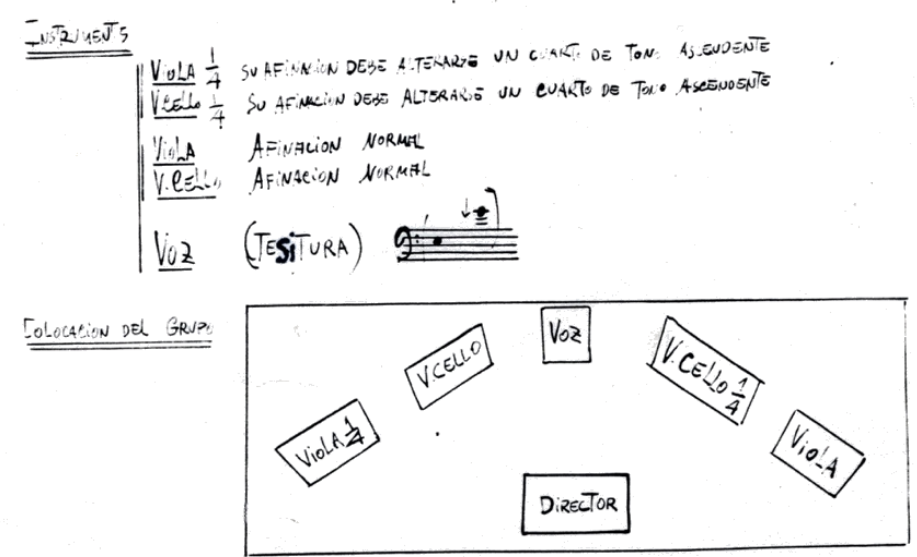

Fig.4

La voz tiene un tratamiento instrumental, ateniéndose siempre a la sonoridad fonética del texto, de manera que se tenga presente la base de articulación de la lengua francesa en su doble aspecto de base tensa y creciente: «mode tendu» $\mathrm{y}$ «mode croissant». El primer análisis realizado en el CSIC ofreció una información gráfica bastante limitada, teniendo en cuenta la simplicidad de un medio que entonces se consideraba muy moderno pero cuyas funciones eran asimismo reducidas. Un sencillo espectrógrafo (fig. 5) que disponía asimismo de un alimentador, un amplificador y motor de rotación; un micrófono (M), conmutador (C), disco magnético (D), filtro (F), aguja inscriptora (A), cilindro reproductor $(\mathrm{CR})$, eje $(\mathrm{E})$ y corredera $(\mathrm{d})$. 
La música pentagramada aparece a lo largo de la partitura solamente de forma ocasional, pues las técnicas empleadas demandan una notación específicamente concebida para su realización. Cinco son los tipos de grafías empleadas, adaptadas a otros tantos elementos fonéticos: 1) grafías correspondientes a la estructura tonal de cada frase y entonación de cada grupo fónico; 2) grafías para la diferenciación de los espacios entre grupos fónicos de menor a mayor (], ]], ]]], etc.); 3) indicación de los grupos fónicos rítmicamente más importantes en cuanto a posible ritmo total del texto, esto es, en

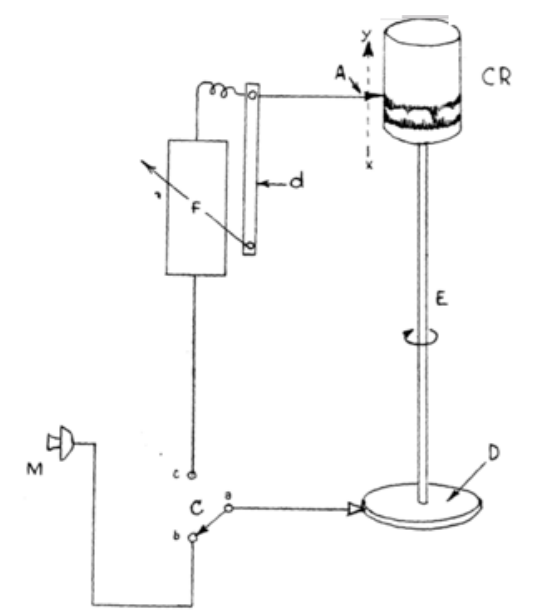

Fig. 5 cuanto a su posición dentro del mismo; 4) grafías correspondientes a los tonemas con sus cinco diferentes terminaciones: cadencia, anticadencia, semicadencia, semianticadencia y suspensión; 5) grafías que señalan la acentuación máxima de la estructura tonal de cada frase, también en escala de menor a mayor (>, >>, > >, etc.) y las que indican matiz de acentuación-duración ( \langle\rangle$,\langle\rangle\langle\rangle,\langle\rangle\langle\rangle\langle\rangle$, etc.). La elección de estos signos no es arbitraria en ningún caso; en su mayoría están tomados del propio análisis espectrográfico y su rigor técnico quedó supervisado por Quilis y por la documentación bibliográfica específica que sirvió de soporte al compositor. Para el estudio de las vocales, Acilu toma como referencia el estudio de Delattre (1948) en el que se sitúan las once vocales orales francesas a partir de los resultados ofrecidos por el espectrógrafo. La función principal de la primera parte de la obra es el acercamiento del valor semántico de las palabras de Teilhard de Chardin al auditorio, un valor que ya no volverá a aparecer en las restantes secciones de la obra. El análisis de la lectura completa del texto, realizado por Quilis, proporcionó un primer documento base sobre el que trabajar de manera muy rudimentaria (fig. 6). 


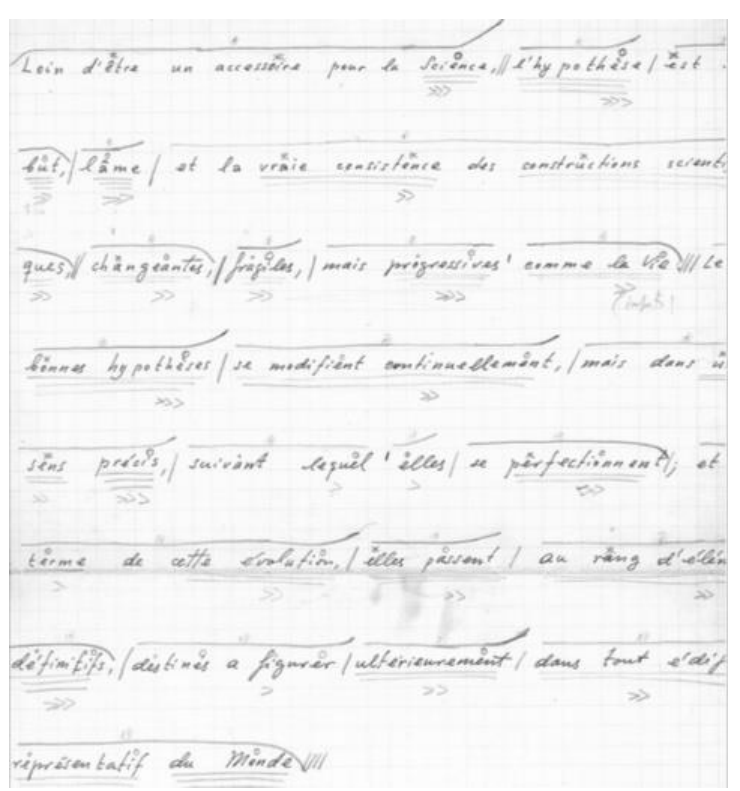

Fig. 6

En la actualidad las nuevas tecnologías ponen a disposición de los investigadores medios que no precisan del laboratorio de fonética. Paul Boersma y David Weenik (2019) desarrollaron en la Universidad de Amsterdam un programa multiplataforma, de código abierto, denominado PRAAT. Se trata de una herramienta de trabajo fundamental a libre disposición de los usuarios desde inicios de los años noventa del siglo pasado, que va implementando sus avances y posibilidades de manera progresiva. El programa, que está diseñado específicamente para las investigaciones en el ámbito de la fonética, permite realizar análisis de textos desde dispositivos personales: solamente se precisa un ordenador y un micrófono de entrada de sonido. Una aplicación moderna como Shazam ${ }^{31}$ identifica fragmentos sonoros mediante un catálogo de huellas digitales de audio basadas en el espectrograma, de forma que las consecuencias de los primeros experimentos de análisis fonético han llevado a fines impensables en su origen. Los medios técnicos actuales habrían ayudado muchísimo a la realización más precisa del texto de Pierre Teilhard de Chardin, como puede observarse en este análisis espectrográfico del texto completo llevado a cabo con el programa PRAAT (fig. 7). En este análisis se advierte cómo el tratamiento específico de la voz en las cuatro secciones principales en que puede estructurarse la obra se ha realizado teniendo en cuenta que la articulación de los sonidos se ha interpretado según su entonación hablada, no cantada ${ }^{32}$.

\footnotetext{
${ }^{31}$ Shazam se presentó en 2008 en versión para iPhone 2.0. y en 2009 ya tenía diez millones de usuarios. Fue adquirido por Apple en 2017. Su funcionamiento es como un espectrógrafo portátil, si bien necesita una base de datos previos, que es la que compara y distingue el patrón de cada composición mediante el algoritmo de la aplicación.

${ }^{32}$ Lectura del texto realizada por el Dr. D. Francisco González Fernández (hablante bilingüe francés-español), Catedrático de Filología Francesa de la Universidad de Oviedo. Instalación de PRAAT para Mac: Dra. Dña. Carmen Muñiz, profesora del departamento de Filología Española de la Universidad de Oviedo. La sección final, indicada por la barra de desplazamiento vertical, corresponde al nombre del autor del texto, «Pierre Teilhard de Chardin», que se pronuncia tras una breve pausa una vez finalizada la lectura del mismo.
} 


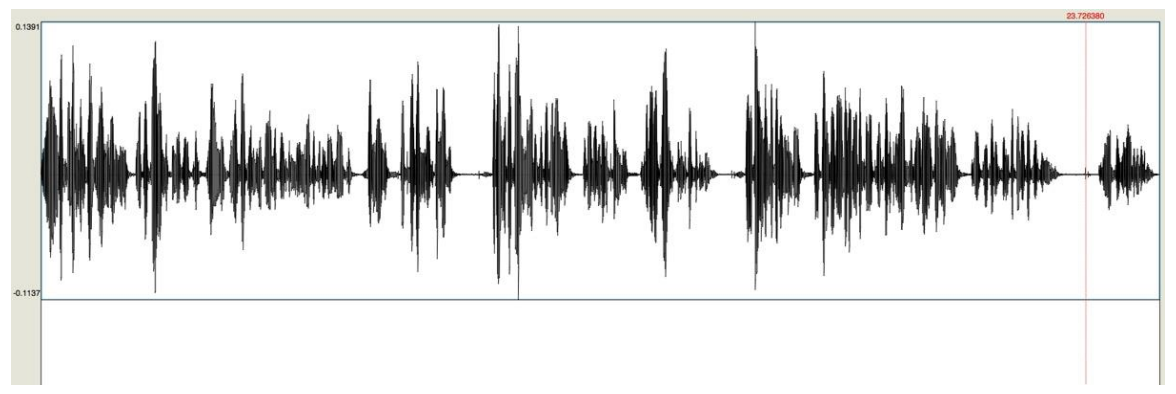

Fig. 7

En la primera sección el intérprete lee el texto completo en la altura que resulte más cómoda a su tesitura, pero poniendo cuidado con el tono de su voz, que debe evidenciar las cadencias y anticadencias allí donde las respectivas grafías se lo indican y teniendo en cuenta las escalas de intensidades y vibración en cada una de ellas, así como la separación de grupos fónicos y la acentuaciónduración. La lectura debe ceñirse a la semiografía del texto, a los signos escritos siguiendo la velocidad de la palabra. Frente a la simplicidad de medios gráficos empleados en la partitura original (fig.8), se observa la precisión del análisis de ese mismo tramo sonoro mediante PRAAT (fig.9), que refleja con exactitud la altura, tono e intensidad de los formantes seleccionados. El material instrumental de esta primera parte actúa como fondo mutable que en ocasiones coincide con los espacios de separación de los grupos fónicos, es decir, con la proporción entre dos pausas. La voz está escrita en una sola línea que corresponde al registro hablado. Esta sección inicial permite acercar el valor semántico del texto al auditorio por primera y única vez.

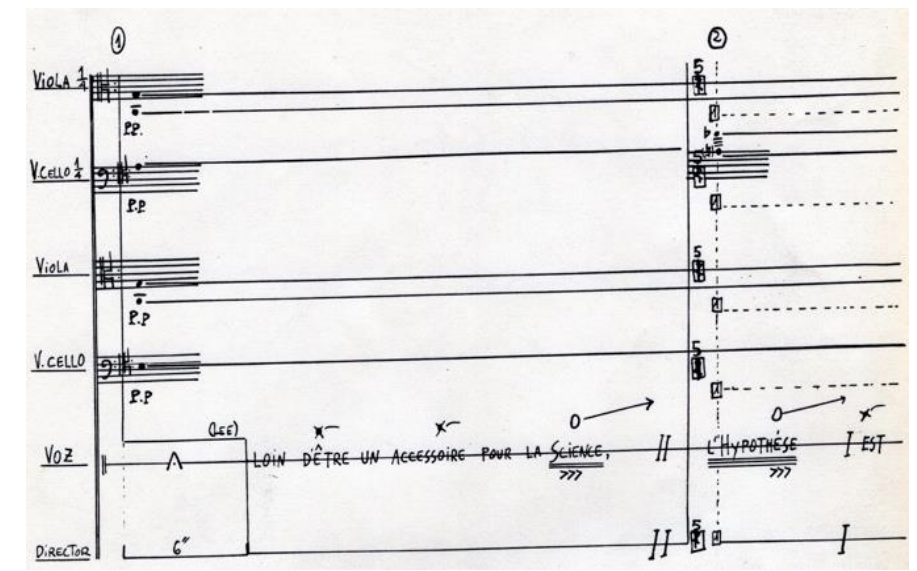

Fig. 8 


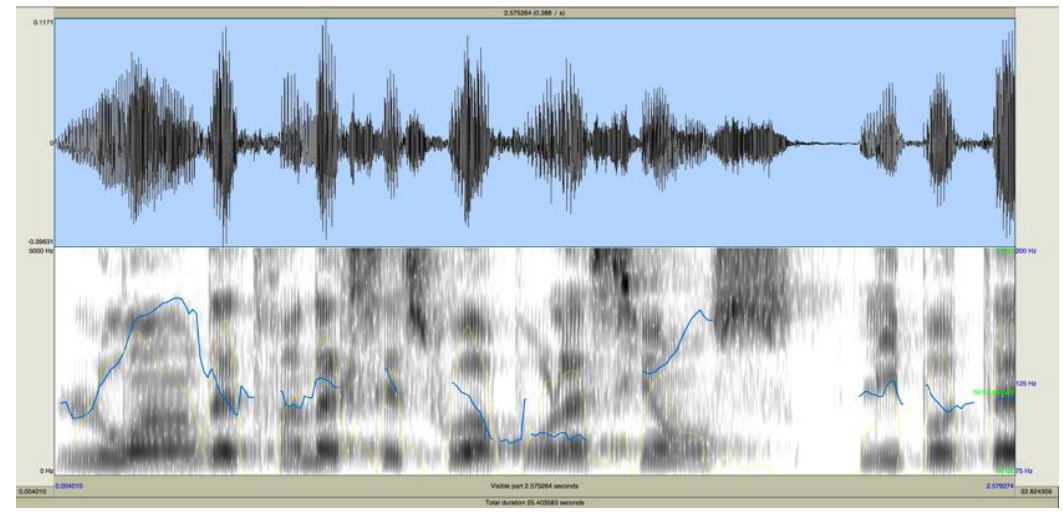

Fig. 9

La tesitura de la voz se divide entre los compases [6-40] en cuatro partes escritas en tetragrama, empleando intervalos irregulares o «no fijos»: de las cuatro líneas, la primera corresponde a la altura más cómoda para el solista vocal, es decir, la articulación hablada. La tesitura se mantiene así dividida en cuatro alturas no temperadas que derivan de la propia dinámica (fig10).

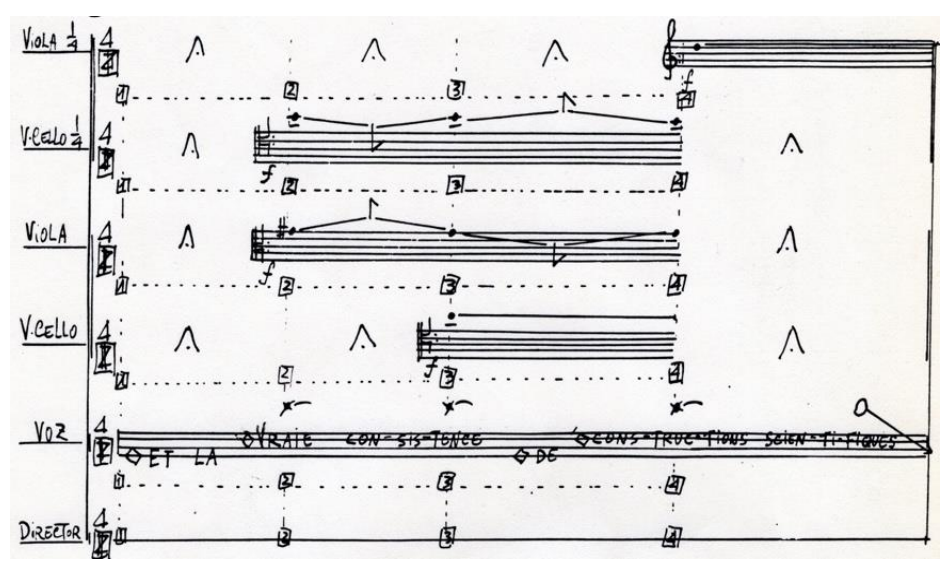

Fig. 10

Más adelante será necesario pasar a la escritura en hexagrama, una grafía que procede de la necesidad de crear cinco espacios a los que se suma la línea correspondiente a la articulación hablada (fig.11). 


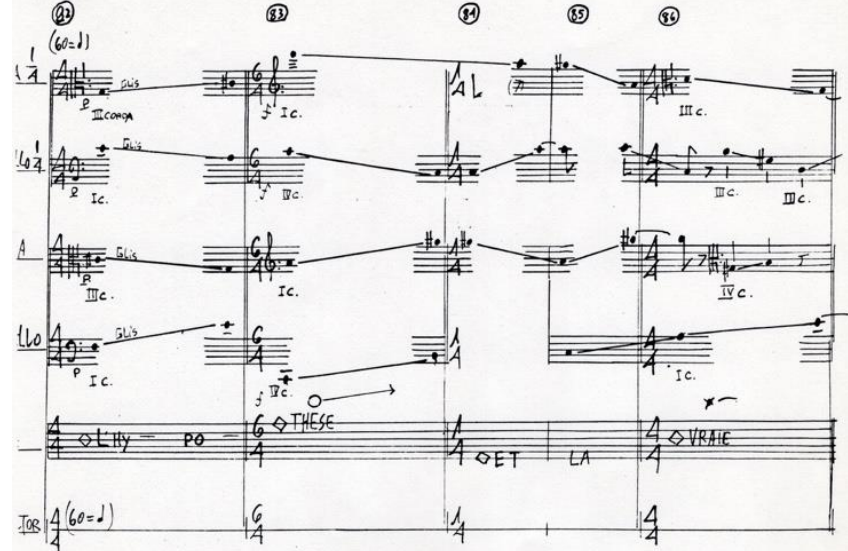

Fig. 11

Técnicamente es importante el hecho de que la pronunciación de las vocales en esta sección final sea la del español, ya que, frente a los criterios elegidos por Acilu en las secciones anteriores, tales como la mayor modulación que presenta la lengua francesa comparada con otras (como la española o la italiana) o el hecho de que su base de articulación posea una tensión mayor, en esta última parte se inclina a subrayar los rasgos de brevedad, claridad y precisión que caracterizan a las vocales españolas y que dan firmeza a nuestro sistema vocálico, como señala Navarro Tomás en su discurso de ingreso en la Real Academia Española, siguiendo la descripción de Storm al comparar la entonación española con la italiana haciendo notar que «mientras esta última, con sus vivas y amplias ondulaciones, recorre toda la escala musical, la cadencia del castellano, más uniforme, severa y mesurada, se concentra en formas precisas» ${ }^{33}$. Los compases finales concentran el mayor rendimiento del material vocálico poniendo de manifiesto el valor musical que poseen las unidades fónicas más simples de acuerdo con los criterios de clasificación acústica, orden de perceptibilidad (fig.12) y orden de frecuencia (fig.13).

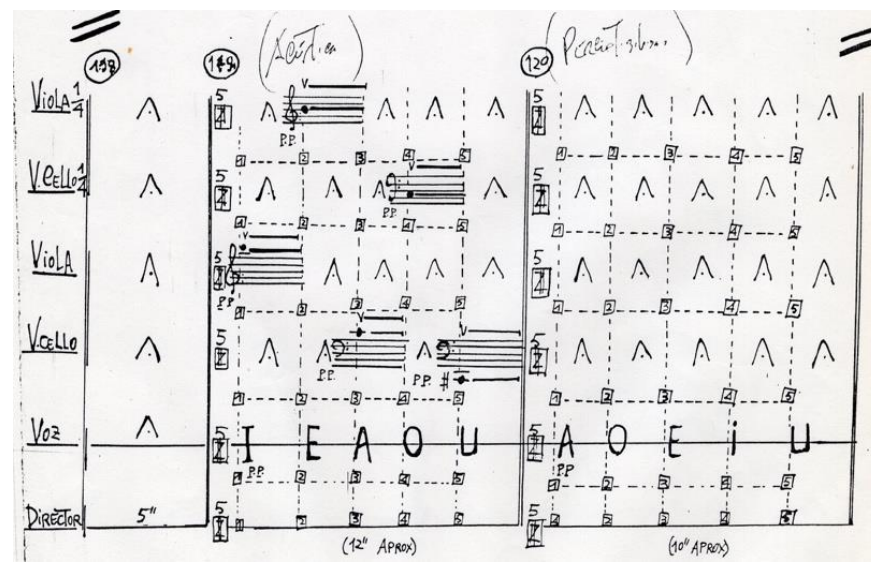

Fig.12

\footnotetext{
33 «El acento castellano », es el título con el que Navarro Tomás rotuló su discurso en el acto de su recepción académica el día 19 de mayo de 1935. Menciona en dicho texto a J. M. Storm, citando sus propias palabras de una publicación muy anterior, «Romanische Quantität» (Phonetische Studien, 1889). En cuanto a la lengua francesa, se refiere a ella en este mismo discurso ensalzando cómo « el francés labra y perfila con esmero sonidos, sílabas e inflexiones », y «al francés se le elogia por su forma conversable, refinada y flexible ».
} 
escuchó por primera vez en la voz de José Luis Ochoa de Olza y el Grupo Alea dirigidos por Enrique García Asensio ${ }^{34}$.

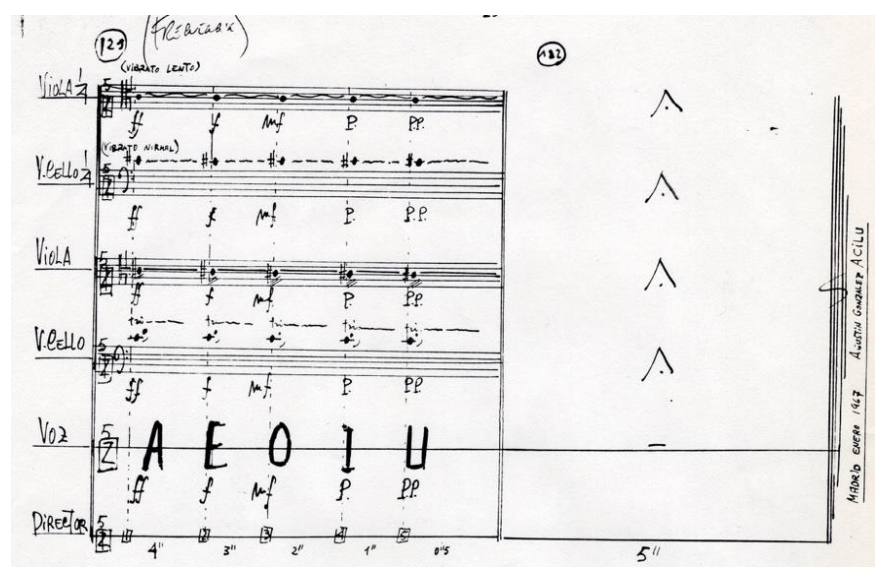

Fig. 13

\section{Conclusiones}

En las dicotomías aquí planteadas - prosa y poesía, arte y ciencia, música y fonética- cobordismos y límites se plantean como hipótesis, bien para demorarse en sus contornos confiando en que la especulación ofrezca resultados inéditos, bien para ser traspasados, diluyendo así la demarcación de ámbitos en apariencia extraños. El cobordismo no deja de ser, desplazado del contexto matemático hacia los confines de otras artes y otras ciencias, una metáfora que sirve para explicar algunos intereses comunes y metodologías de trabajo afines a todas ellas. Y el análisis, como sostiene Baudrillard en Le trompe-l'œil, un pretexto para reflexiones respecto a posibles teorías filosóficas o nuevas vías de indagación epistemocrítica. Ámbito lingüístico y dominio musical resultan complementarios, tal como acabamos de ver en el análisis de Dilatación fonética. Abandonada la idea de supeditar el texto a una sonorización extraña, por bien que esta suene al oído, el contenido semántico del mismo resulta absolutamente indiferente a efectos musicales. Probemos a escuchar en versión original A kékszakállú herceg vára -El castillo del duque de Barbazul- de Bèla Bartók, donde la historia narrada, por conocida, carece de intriga e interés. Para un oyente no hablante del idioma húngaro la musicalidad de la lengua magiar se impone sobre cualquier otra melodía.

El pensamiento teilhardiano, síntesis de principios que asumen ciencia, técnica y progreso en el contexto de una significación espiritual, padeció en vida la incomprensión personal y profesional de parte de ambas facciones -filosofía y ciencia- para gozar «à titre posthume d'une fortune intellectuelle peu commune» (Escalère, 1967: 192). El pensamiento sonoro de González Acilu, tan innovador desde sus primeras páginas dedicadas a la especulación sonora a partir de la

${ }^{34} \mathrm{Su}$ estreno tuvo lugar en el Salón de Actos del INP de Madrid el 24 de mayo de 1968. La obra está dedicada «A la memoria de Pierre Teilhard de Chardin». 
lengua, encaja en la conjunción ciencia-arte que plantea Teilhard de Chardin y asume, por si fuera poco, la hipótesis como razón única de discurso a lo largo de una producción sonora que se extiende desde los años sesenta hasta hoy.

Fundador de la topología diferencial con su teoría del cobordismo, René Thom disfrutaba con la matemática, que tantas veces definió como un campo de fronteras móviles y abiertas, un lugar en el que existe una zona de conocimiento donde todavía se pueden experimentar verdaderas maravillas. René Thom, Teilhard de Chardin y González Acilu desarrollan sus ideas en los límites topográficos de ámbitos distintos, se mueven con desenvoltura entre haces de isoglosas. Disfrutan de una libertad casi furtiva experimentando los intercambios más intensos en los espacios limítrofes. Ponen un pie a cada lado de la línea divisoria, atraviesan de un lado a otro con naturalidad seguros, como lo estaba Kapucinski, de que el sentido de la vida es cruzar fronteras.

Poincaré, como ya hemos mencionado antes, consideraba que a menudo la hipótesis es no solo necesaria, sino legítima. Añadimos a sus palabras una reflexión del mismo texto de Bataille citado al inicio de estas páginas: con frecuencia la transgresión es algo admitido, e incluso prescrito.

\section{REFERENCIAS BIBLIOGRÁFICAS}

BARKER, Stephen Francis (1957): Induction and Hypothesis. A Study of the Logic of Confirmation. Nueva York, Cornell University Press.

BARTHES, Roland (1984): «De l' œuvre au texte», in Le bruissement de la langue. París, Éditions du Seuil, 61-110.

BarthÉLEMY-Madaule, Madeleine (1963): Bergson et Teilhard de Chardin. París, Éditions du Seuil.

BARTHÉLEMY- MADAUle, Madeleine (1967): Bergson. París, Éditions du Seuil.

BATAILlE, Georges (1965): L'Érotisme. París, Les Éditions du Minuit.

BAUdelaIRE, Charles (1920 [1862]): Petits poèmes en prose. París, Helleu et Sergent.

BAUdelaIRE, Charles (1869): «Petits poèmes en prose», in Euvres completes IV. París, Michel Lévy Frères.

BERNARD, Suzanne (1959): Le poème en prose. De Baudelaire jusqu'à nos jours. París, Nizet.

Boersma, Paul \& David WeEnIK (2019): Praat: Doing Phonetics by Computer. Amsterdam, University of Amsterdam.

Bourguignon, Jean-Pierre (2004): «René Thom: Mathématicien et apprenti philosophe ». Bulletin of the American Mathematical SocietK, 41, 273-274.

Bowen, Zack (1995): «The Bronzegold Sirensong: A Musical Analysis of the Sirens Episode in Joyce's Ulysses», in Zack Bowen (ed.), Bloom's Old Sweet Song : Essays on Joyce and Music. Tampa, University Press of Florida, 25-76.

Braithwaite, Richard B. (1965): Scientific Explanation. A Study of the function of theory, probability and law in science. Cambridge, Cambridge University Press. 
BREMOND, Henri (1926): La poésie pure. Avec un débat sur la poésie, par Robert de Souza. París, Grasset.

CALABRESE, Omar (1987): El lenguaje del arte. Barcelona, Paidós.

CARNERO, Guillermo (1989): Las armas abisinias. Ensayos sobre literatura y arte del siglo XX. Barcelona, Anthropos.

CARNERO, Guillermo (2010): «Arácnido confuso: purismo y neogongorismo en el primer Miguel Hernández», in José Carlos Rovira \& Carmen Alemany (coord.), Miguel Hernández. La sombra vencida. Madrid, Sociedad Estatal de Conmemoraciones Culturales.

CuÉNOT, Claude (1967): Pierre Teilhard de Chardin. Las grandes etapas de su evolución. Madrid, Taurus.

CuReses, Marta (2001): Agustín González Acilu. La estética de la tensión. Madrid, ICCMU [1 $1^{\mathrm{a}}$ ed.: 1995].

CURESES, Marta (2011): «González Acilu. Ética y estética del límite». Revista Internacional de Estudios Vascos, 56:I, 54-106.

DELATTRE, Pierre (1948): «Un triangle acoustique des voyelles orales du français ». The French Review, XXI:6, 447-484.

Delattre, Pierre (1958): «Les indices acoustiques de la parole». Phonetica, 2, 226-251.

DeluY, Henry (1989): Poésie en France (1983-1988). Une anthologie critique. París, Flammarion.

ECO, UMBERTO (1979): Obra abierta. Barcelona, Ariel.

ESCALÈRE, Bernard (1967): «À partir de Teilhard de Chardin ». Cahiers du CEDIAS (Centre d'Études de Documentation, d'Information et d'Actions Sociales), 5, 191-206.

Garaudy, Roger (1959): Perspectives de l'homme. Existentialisme, pensée catholique, marxisme. París, Presses Universitaires de France.

GONZÁlEZ FERNÁNDEZ, Francisco (2012): Esperando a Gödel. Literatura y matemáticas. Madrid, Nivola.

GUIETTE, Robert (1964): «Baudelaire et le poème en prose». Revue belge de Philologie et d'Histoire, 42-43, 843-852.

HAYEK, Nácere (2006): «Una biografía de René Thom ilustrada con comentarios sobre su vida y su obra científica». Matematicalia 2, 4. URL: http://www.matematicalia.net/index.php?option=com_content \&task=view\&id=289\&Itemid=186.

Hugo, Victor (1971 [1827]): Manifiesto romántico. Introducción de Henri de SaintDenis. Traducción de Jaume Melentres. Barcelona, Ediciones Península.

KAHN, Gustave (1897): Premiers poèmes. París, Mercure.

KaHN, Gustave (1925): Charles Baudelaire. Son æeuvre. París. Éditions de la Nouvelle Revue Critique.

LÉVI-STRAUSS, Claude (1970): «Las matemáticas del hombre», in VVAA, Estructuralismo y epistemología. Buenos Aires, Ediciones Nueva Visión.

MALMBERG, Bertil (1952): «Le problème du classement des sons du langage». Studia Linguistica, VI, 1-56. 
MALMBERG, Bertil (1956): «Questions de méthode en phonétique syncronique». Studia Lingüística, X, 1-44.

NAVARRo ToMÁs, Tomás (1960): «El método espectrográfico. Notas de fonética experimental». Revista de Filología Española, XLIII:3-4, 415-428.

NAVArro Tomás, Tomás (1968-1969): «Don Ramón Menéndez Pidal en el Centro de Estudios Históricos». Anuario de Letras, VII (Homenaje a Menéndez Pidal), 924.

ORTEGa y GASSET, José (1990 [1914]), Meditaciones del Quijote. Edición de Julián Marías. Madrid, Ediciones Cátedra.

PAQUIN, Jacques (2008): «L'intertexte scientifique en poésie». Épistemocritique. Revue de littérature et savoirs, 3. URL: https://epistemocritique.org/lintertextescientifique-en-poesie.

PATER, Walter (1988 [1877]): «The School of Giorgione», in The Renaissance : Studies in Art and Poetry. Oxford, A. Philips Oxford University Press.

PetrTot, Jean (1983), «Sémiotique et théorie des catastrophes». Actes sémiotiques, 47$48,4-64$.

PETITOT, Jean (2015): «Les premiers textes de René Thom sur la morphogénèse et la linguistique : 1966-1970». HAL. URL: https://hal.archives-ouvertes.fr/hal$01265180 \mathrm{v} 2$.

PETITOT, Jean (sd): «Théorie des catastrophes», in Encyclopaedia Universalis [en línea]. URL: https://www.universalis.fr/encyclopedie/theorie-des-catastrophes.

POE, Edgar Allan (1846): «The Phylosophy of Composition». Graham's Magazine, XXVIII 4-28, 163-167.

POINCARÉ, Henri (1968): La Science et l'hypothèse. París, Flammarion.

QUILIS, Antonio (1960): «El método espectrográfico. Notas de Fonética experimental». Revista de Filología Española, XLIII, 3-4.

QuILIS, Antonio (1964): Curso de fonética y fonología. Madrid, CSIC.

RESCHER, Nicholas (1981): Sistematización cognoscitiva. Madrid, Siglo XXI Editores.

Robinson, Judith (1962): L'analyse de l'esprit dans les Cahiers de Valéry. París, Corti

SANDRAS, Michel (1995): Lire le poème en prose. París, Dunod.

SALANSKIS, Jean-Michel (2019): «Épistémologie de la mathématique. Théorie des catastrophes», in Encyclopadia Universalis [en línea]. URL: https://www.universalis.fr/encyclopedie/epistemologie-de-la-mathematique/6.

SCHWARTZ, Gustavo Ariel \& Eduardo BERTI (2018): «Literatura y ciencia. Hacia una integración del conocimiento». Arbor, 194 (790). URL: https://doi.org/10.3989/arbor.2018.790n4006.

SOURIAU, Maurice (1897): La Préface de Cromwell. Introduction, texte et notes. París, Société Française d'Imprimerie et de Librairie.

STEINER, George (1990): Lecturas, obsesiones y otros ensayos. Madrid, Alianza.

TEILHARD DE CHARDIN, Pierre (1925): «L'histoire naturelle du monde. Reflexions sur la valeur et l'avenir de la systématique». Scientia : Rivista internazionale di sintesi scientifica, XXXVII:1, 15-24. 
TEILHARD DE CHARDIN, Pierre (2017): La vida cósmica. Escritos del tiempo de la guerra (1916-1917). Madrid, Trotta.

Thom, René (1971): Modèles mathématiques de la morphogénèse. París, Christian Bourgois.

THOM, René (1972): Stabilité structurelle et morphogenèse. Essai d'une Théorie générale des modèles. Nueva York, Benjamin.

THOM, René (1985): Parábolas y catástrofes. Entrevista sobre matemática, ciencia y filosofía a cargo de Giulio Giorello y Simona Morini. Barcelona, Tusquets.

THOM, René (1988): Esquisse d'une Sémiophysique. Physique aristotélicienne et Théorie des Catastrophes. París, InterÉditions.

UTRERA, María Victoria (1999): Teoría del poema en prosa. Sevilla, Universidad de Sevilla.

VESCIA, Remo (2015): «Pierre Teilhard de Chardin, prêtre, savant, poète et visionnaire», in Exposition «Ensemble, construisons la Terre». URL: https://www.teilhardinternational.com/assise2010/actualité/pierre-teilhard-de-chardin-pretre-savantpoete-et-visionnaire-prophete-de-lesperance.

WhEWELL, William (1984): Selected Writings on the History of Science. Edición de Yehuda Elkana. Chicago, The University of Chicago Press.

Whewell, William (1989): Theory of Scientific Method. Edición de Robert E. Butts. Indianápolis-Cambridge, Hackett Publishing.

WILDGEN, Wolfgang (1999): De la grammaire au discours. Une approche morphodynamique. Berlín, Peter Lang. 